\title{
Distributions qualitative et quantitative des éléments nutritifs dans un jeune peuplement de Pin maritime (Pinus pinaster Ait)
}

\author{
B. LEMOINE, J. RANGER *, J, GELPE \\ Avec la collaboration technique de : \\ Louisette GELHAYE *, Dominique RANGER *, M. ADRIAN *, \\ J.-P. BOIREAU, D. BOUCHARD *, \\ M. GUEDON, B. LAFON $\uparrow$, Y. LEFEVRE *, G. LEFROU, \\ J.-PH. ROSSETTO et A. SARTOLOU \\ INRA, Laboratoire de Sylviculture et d'Ecologie \\ Centre de Recherches de Bordeaux, Pierroton, F 33610 Cestas \\ * INRA, Station de Recherches sur le Sol, la Microbiologie \\ et la Nutrition des Arbres forestiers, \\ Centre de Recherches de Nancy, Champenoux F 54280 Seichamps
}

\section{Résumé}

Le travail présenté ici fait suite à un article publié dans cette revue (Lemorne et al., 1986) et traitant de la distribution de la matière sèche dans un jeune peuplement de Pin maritime situé dans la zone humide des Landes de Gascogne. Ces nouveaux résultats concernent l'analyse de la distribution des éléments nutritifs majeurs (N, P, K, Ca, Mg) dans le même peuplement.

Les principaux résultats concernent :

- la distribution qualitative (concentration) des éléments dans les différents compartiments L'analyse statistique (A.F.D.) permet d'établir pour la couronne et le tronc des profils synthétiques biologiquement interprétables qui rendent compte des variations majeures des concentrations. nelle

Ces profils seront modélisés mathématiquement et feront l'objet d'une interprétation fonction-

Outre les caractéristiques moyennes, les variations individuelles ont été étudiées. Les tests de similarité montrent que cet effet n'est pas identique pour la couronne et pour le tronc et, par conséquent, n'est pas contrôlé par les mêmes facteurs pour les différents compartiments.

L'évaluation de la minéralomasse du peuplement montre que le Pin maritime possède un rendement élevé des éléments biogènes ; la frugalité de cette espèce est la plus nette pour le calcium et le phosphore.

Au plan méthodologique, l'échantillonnage utilisé conduit à une estimation fiable des minéralomasses (erreur à craindre inférieure à $10 \mathrm{p}$. 100). Il ne semble pas possible de le réduire significativement si l'on conserve le double objectif qualitatif et quantitatif.

Les lois de variation des bioéléments mises en évidence dans ce jeune peuplement devront être vérifiées dans d'autres peuplements et dans d'autres conditions écologiques.

Mots clés : Pinus pinaster Ait., éléments nutritifs, concentration des tissus, minéralomasse. 


\section{Introduction}

Le Pin maritime des Landes de Gascogne constitue un système écologique artificiel d'origine récente et d'amplitude géographique unique en France (environ 1 million d'hectares). La ligniculture permet une production élevée $\left(12 \mathrm{~m}^{3} \cdot \mathrm{ha}^{-1} \cdot \mathrm{an}^{-1}\right.$ en moyenne) sur des sols très pauvres avec certes un apport d'éléments par fertilisation, au moins en lande humide (GELPE \& LeFrou, 1986).

Le but des études que nous avons entreprises dans cet écosystème est de caractériser les principaux paramètres du cycle biogéochimique de façon à connaître les besoins exacts de l'espèce et de montrer les limites écologiques de ce système aux sollicitations multiples. En effet, le maintien de la productivité des sols soumis à la monoculture risque de se poser dans les mêmes termes qu'en Australie par exemple avec la monoculture de Pinus radiata (Farrel et al., 1986). Les premières études ont porté sur la lande humide (dans le sens défini par Saint-Didier, 1976). Les résultats exposés ici concernent :

- l'analyse biométrique de la distribution des bioéléments dans un jeune peuplement de Pin maritime,

- la quantification des minéralomasses compartimentées des arbres et du peuplement

\section{Matériel et méthodes}

\subsection{Le site : peuplement et sol}

L'étude porte sur un peuplement artificiel de Pin maritime (Pinus pinaster Ait.) de 16 ans qui a subi un traitement sylvicole classique de la pratique landaise. Il s'agit en fait d'un peuplement composite, résultant de la réunion de deux répétitions d'un traitement d'une expérience de fertilisation installée au lieu dit Berganton, sur la commune de Saint-Jean-d'Illac en 1962, dans le faciès humide des Landes de Gascogne (Comps et al., 1979). Une analyse statistique en composantes principales portant sur six caractères dendrométriques mesurés sur 30 arbres dans chaque placeau (circonférences et hauteur du tronc, longueur et largeur de la cime) a montré la grande ressemblance des deux placeaux. Ceci nous a permis de répartir l'échantillonnage destructif de façon à ne pas compromettre l'avenir de l'expérience.

Les caractéristiques du peuplement (moyenne des deux placeaux) sont les suivantes :

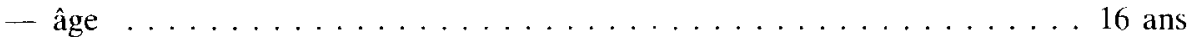

- hauteur dominante ..................... 13,30 m

- hauteur moyenne . . . . . . . . . . . . . . 12,30 m

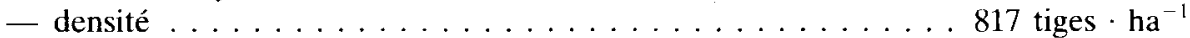

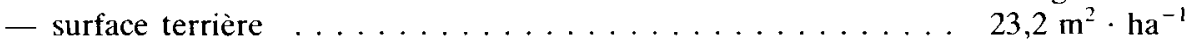

— circonférence de l'arbre de surface terrière moyenne . . . . . . 59,7 cm

Ce peuplement appartient à la classe I de la table de production établie pour les peuplements des Landes de Gascogne (Decourt-Lemoine, 1969). 
Le climat général est de type atlantique avec une pluviométrie moyenne annuelle de $950 \mathrm{~mm}$ et une température moyenne annuelle de $12,8^{\circ} \mathrm{C}$. Le sol, développé sur les dépôts détritiques sableux du Miopliocène décrits par LE Gigan (1979), est un podzol humique hydromorphe présentant le plus généralement un alios (B spodique induré) vers $60 \mathrm{~cm}$ de profondeur. Cet horizon constitue une limite physique (induration) et/ou chimique (toxicité aluminique) à l'enracinement. Pendant la période hivernale, le niveau supérieur de la nappe phréatique atteint fréquemment l'horizon superficiel (Ap). La matière organique est peu évoluée (acidité et hydromorphie en sont la cause), l'humus est un mor au $\mathrm{C} / \mathrm{N}$ de l'ordre de 25 (RIGHI, 1979). Le $\mathrm{pH}$ du sol, voisin de 4 en Ap Al, est un peu plus élevé en profondeur (5).

La capacité d'échange cationique ne dépasse pas $5 \mathrm{meq} / 100 \mathrm{~g}$ dans les horizons organiques, elle n'est que de $1 \mathrm{meq}$ dans la partie minérale du profil avec un taux de saturation inférieur à $10 \mathrm{p} .100$ provenant du calcium et du magnésium. Les réserves en éléments nutritifs sont très faibles, même en tenant compte des apports par fertilisation : 140 unités de phosphore $\left(\mathrm{P}_{2} \mathrm{O}_{5}\right)$ en 1963 et 90 unités en 1974 ont été apportées sous forme de scories de déphosphoration puis de phosphates naturels broyés. L'apport indirect de chaux est élevé $\left(400 \mathrm{~kg} \cdot \mathrm{ha}^{-1}\right)$.

La végétation concurrente du peuplement forestier est essentiellement composée de Molinie (Molinia coerulea Moench.), d'avoine de Thore (Pseudoarrhenaterum longifolium Thor.), de brande (Erica scoparia L.), d'ajoncs (Ulex nanus $\mathrm{Sm}$., Ulex europaeus L.), de Bourdaine (Rhamnus frangula L.).

Cette végétation est régulièrement détruite par scarification sur la moitié de la surface cadastrale des peuplements (interbande). Sa production annuelle est de 0,31 tonne de matière sèche par hectare dans ce site.

\subsection{Echantillonnage}

L'échantillonnage concernant les biomasses a porté sur 20 arbres distribués dans les deux placeaux. Il est de type stratifié (SAToo, 1982) sur la circonférence à $1,30 \mathrm{~m}\left(\mathrm{C}_{130}\right)$ des individus. Il a eu lieu en mars 1979. L'échantillonnage concernant les minéralomasses a porté sur 11 des 20 arbres.

Sur chaque arbre, les mesures suivantes ont été effectuées :

— circonférence à la souche $(\mathrm{Cs})$, à $1,30 \mathrm{~m}\left(\mathrm{C}_{130}\right)$, à mi-hauteur et à la base de la première branche vivante ;

- hauteur totale de l'arbre, hauteur de la première branche vivante, hauteur de chaque verticille principal et secondaire (arbres polycycliques) ;

- diamètre à $10 \mathrm{~cm}$ de l'insertion $\left(\mathrm{D}_{10}\right)$ et orientation de chaque branche ;

- poids humide du tronc total.

Le prélèvement d'échantillons pour la détermination de la matière sèche et de la composition chimique est effectué comme suit :

-- échantillonnage d'un tiers des branches de tous âges (376 au total), mesure de leur longueur, séparation des aiguilles et du bois par pousse annuelle ;

- prélèvement d'une rondelle de tronc par mètre linéaire.

$\mathrm{Au}$ laboratoire, chaque échantillon est séché à $65^{\circ} \mathrm{C}$ afin de déterminer son taux d'humidité ; pour 11 des 20 arbres, les échantillons représentant une branche de chaque 
âge (125) et les rondelles des troncs sont réduites en poudre en vue de l'analyse chimique des éléments majeurs.

\subsection{Analyse chimique des échantillons}

Les méthodes analytiques utilisées sont les suivantes :

- azote $(\mathrm{N})$ : méthode KJELDHALL modifiée

- minéralisation sulfurique en présence de catalyseur au sélénium et sulfate de $\mathrm{K}$;

- dosage colorimétrique à l'autoanalyseur TECHNICON ;

- $\mathbf{P}, \mathrm{K}, \mathrm{Ca}, \mathrm{Mg}, \mathrm{Mn}$ et $\mathrm{Na}$ : minéralisation en voie humide en milieu $\mathrm{H}_{2} \mathrm{O}_{2}+\mathrm{HClO}_{4}$;

- P : dosage colorimétrique sur autoanalyseur TECHNICON du complexe phosphovanadomolybdique pour les teneurs élevées ou dosage colorimétrique manuel du complexe phosphosulfomolybdique pour les faibles teneurs ;

- K, Ca, Mg, Mn et Na : dosage par spectrométrie d'absorption atomique.

\subsection{Méthodes statistiques}

Deux types de méthodes sont employées en utilisant principalement le logiciel mis au point par BARADAT (1980) :

\subsection{L'analyse de régression simple ou multiple}

Elle est utilisée pour la construction de deux types de tarifs permettant de passer, pour les premiers, de la minéralomasse d'un échantillon de branches à celle de la couronne de l'arbre et, pour les seconds, de la minéralomasse d'un compartiment de l'arbre à celle du peuplement entier. Dans le premier cas on utilise l'analyse de covariance qui permet de caractériser l'effet arbre (moyennes ajustées).

La régression simple permet de caractériser la relation minéralomasse-biomasse par compartiments; le modèle allométrique est alors utilisé.

L'utilisation des régressions avec transformation logarithmique de la variable expliquée fournit une estimation biaisée des minéralomasses, que l'on corrige par la méthode de Baskerville (1972).

\subsection{L'analyse factorielle discriminante}

Elle vise à caractériser globalement les variations des concentrations sous l'effet de deux facteurs :

- le facteur position du compartiment dans l'arbre ; il s'agit de la position du verticille pour les branches (en fait de leur âge) et le niveau au-dessus du sol pour le tronc :

- le facteur arbre: dans ce cas, le critère de similarité et la technique du dendrogramme permettent de constituer plusieurs groupes d'arbres au sein desquels l'ensemble des concentrations peut être considéré comme identique. 


\section{Résultats - discussion}

Deux démarches ont été menées : la première répondant à l'objectif principal de l'étude qui est de quantifier les minéralomasses des arbres et du peuplement, la deuxième qui vise à interpréter les variations des concentrations entre les différents organes des arbres mais aussi entre les arbres.

\subsection{Les minéralomasses}

\subsection{Résultats intermédiaires : les branches}

A partir de l'échantillon de branches, on a construit des tarifs destinés à estimer les minéralomasses des couronnes des onze arbres. Trois compartiments sont étudiés: le bois (BR) - regroupant la matière ligneuse, le liber et l'écorce - les bourgeons (T) et les aiguilles (F), et six éléments sont concernés, $\mathrm{N}, \mathrm{P}, \mathrm{K}, \mathrm{Ca}, \mathrm{Mg}, \mathrm{Na}$. On utilise le même modèle que pour les biomasses (Lemoine et al., 1986) avec, pour variables explicatives, le diamètre de la branche $\left(D_{10}\right)$ et son âge $(A)$, soit par exemple pour l'azote dans les aiguilles :

$\log$ minéralomasse $\mathrm{N}$ des aiguilles $=\mathrm{a}_{\mathrm{o}}+\mathrm{a}_{1} \log \mathrm{D}_{10}+\mathrm{a}_{2} \mathrm{~A}+\mathrm{a}_{3} \mathrm{~A}^{2}$

Les résultats de l'analyse de covariance montrent qu'aux effets dominants du diamètre $D_{10}$ et de l'âge $A$ de la branche, se superpose un effet arbre pour la majorité des combinaisons élément-compartiment. Les équations globales (dites régressions intracommunes) nous renseignent sur les caractéristiques moyennes des modèles mais ne peuvent être utilisées sans risquer un biais pour les minéralomasses de certains arbres ; on passe des tarifs moyens aux tarifs d'un arbre, en calculant les constantes $a_{O}$ qui lui sont propres (méthode des moyennes ajustées).

\subsection{Résultats globaux}

On a travaillé à deux niveaux, celui du peuplement et celui des arbres.

\subsection{Niveau peuplement}

Six compartiments ont été individualisés : les aiguilles, les bourgeons, le bois des branches vivantes, le bois et l'écorce du tronc, la biomasse racinaire extraite. Ces six compartiments de l'arbre font l'objet pour chaque élément d'une estimation de leurs minéralomasses par l'intermédiaire d'un tarif à une entrée :

Minéralomasse d'un compartiment $=\mathrm{a}+\mathrm{b} \cdot \mathrm{g}$

(pour un élément donné)

où $\mathrm{g}$ est la surface terrière de l'arbre en mètres carrés. L'application de ces tarifs à l'inventaire des circonférences permet de passer aux minéralomasses des deux placeaux et par extension à celles de l'hectare boisé. Les résultats font l'objet du tableau 1.

Le tableau 2 situe le peuplement de'Pin maritime parmi quelques peuplements de conifères d'âge comparable. 


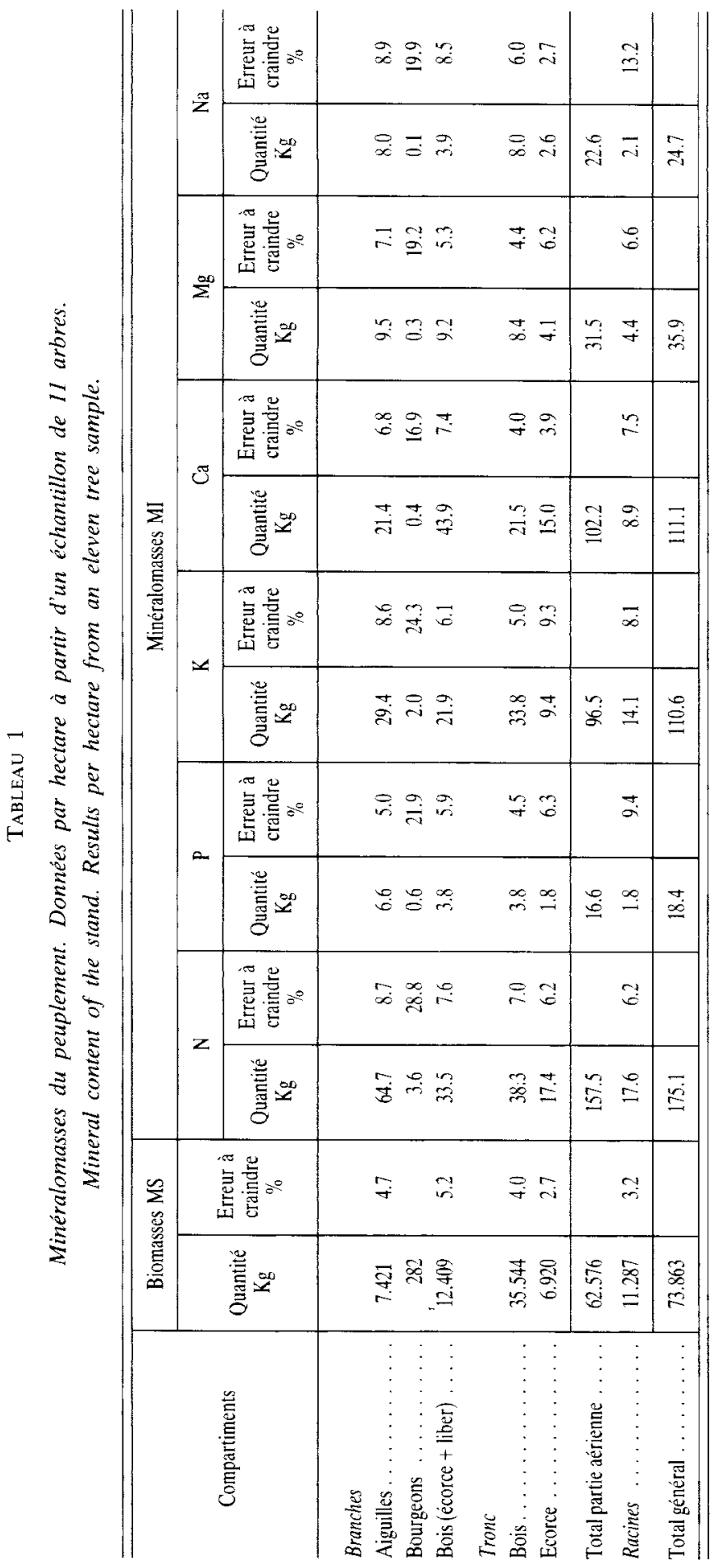




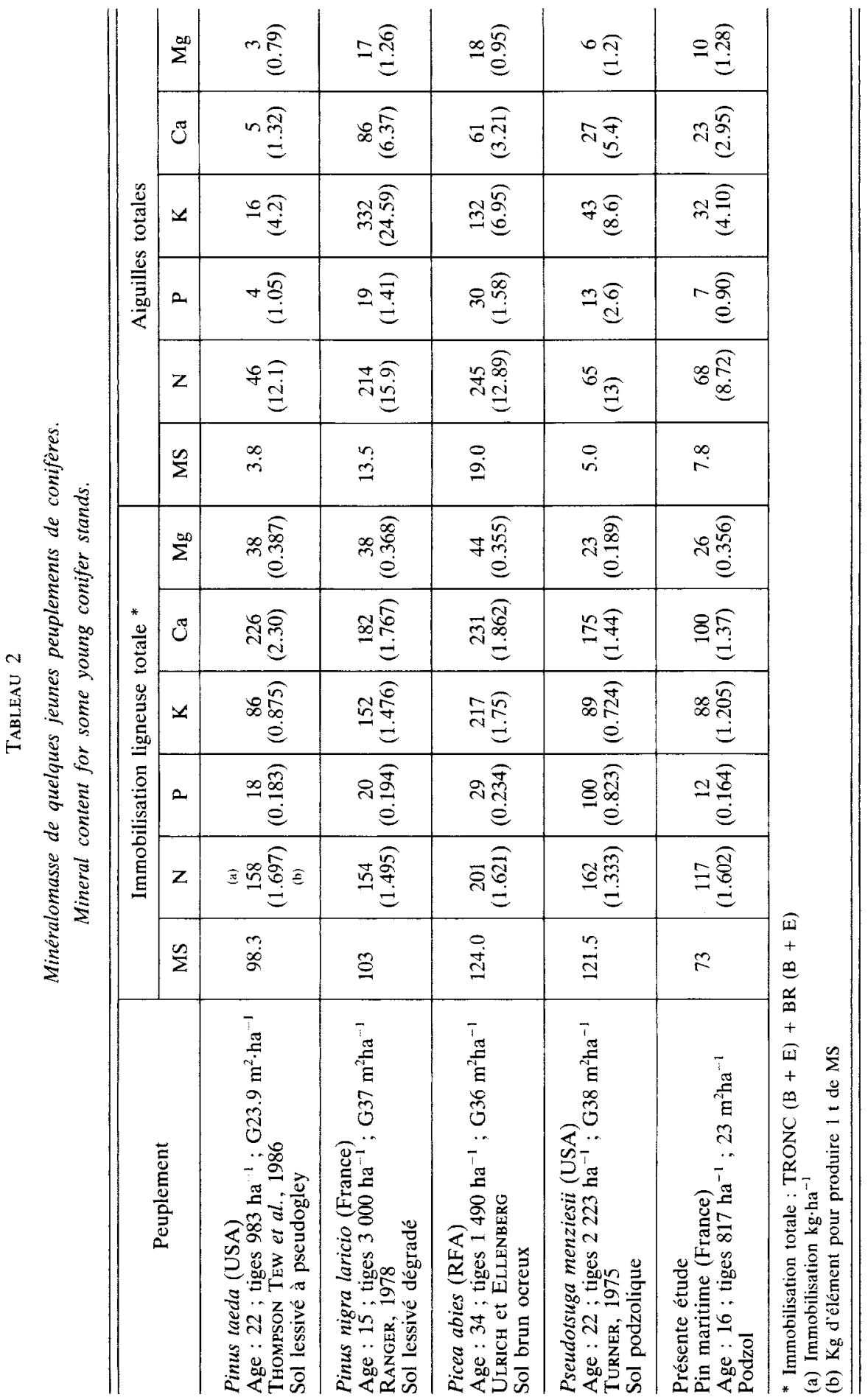


La production (à l'hectare) de biomasse du peuplement de Pin maritime étudiée ici se situe parmi les plus faibles valeurs observées pour les essences classiques de zone tempérée. La ligniculture en est principalement la cause dans la mesure où la moitié de la surface cadastrale est inoccupée (semis en bandes). L'objectif n'étant pas la courte rotation, il est évident que les écarts vont diminuer avec l'âge, comme peut le montrer un calcul approximatif réalisé à partir des tables de production (DEcourT, 1973).

La minéralomasse montre que cette essence est cependant parmi les plus «frugales ». Si l'on compare les immobilisations par tonne de biomasse ligneuse produite, le Pin maritime présente les valeurs les plus basses pour le phosphore et le calcium, ce qui n'est peut-être pas indépendant du facteur sol. Le même calcul effectué pour les aiguilles confirme la frugalité de cette essence avec les teneurs les plus basses pour $\mathrm{N}$, P, K. L'espèce la plus proche serait Pinus taeda L.

\subsection{Niveau arbre}

Il s'agit de savoir pour chaque compartiment si une vigueur accrue de celui-ci, c'est-à-dire de celle de l'arbre auquel il appartient, s'accompagne d'une modification de l'efficience de certains éléments minéraux. Pour cela on examine la relation entre la minéralomasse d'un compartiment et sa biomasse (pour chaque élément). On utilise le modèle d'allométrie afin de tester la proportionnalité entre ces deux caractères. La valeur du coefficient $b$ de la régression :

$\log$ Minéralomasse $=\log a+b \log$ biomasse

est testée par rapport à l'unité.

Le tableau 3 montre que parmi les 30 relations obtenues on n'en trouve que cinq dont le coefficient $b$ diffère significativement de l'unité et seulement trois si on exclut le sodium, élément physiologiquement peu important. Pratiquement, on peut considérer qu'une vigueur croissante s'accompagne :

- d'une concentration croissante du calcium dans les aiguilles; branches ;

- d'une concentration décroissante du magnésium et du phosphore dans les

- d'une concentration constante dans tous les autres cas.

TABLEAU 3

Relations inter-arbres minéralomasse-biomasse.

Relationships between biomass and nutrient content for the eleven trees.

\begin{tabular}{|c|c|c|c|c|c|c|}
\hline & $\mathrm{N}$ & $\mathbf{P}$ & K & $\mathrm{Ca}$ & $\mathrm{Mg}$ & $\mathrm{Na}$ \\
\hline Aiguilles . . . . . . & 1,20 & 1,24 & 1,32 & $1,37 *$ & 1,13 & 0,82 \\
\hline Branches (bois + liber + écorce) & 0,91 & $0,79 *$ & 0,84 & 0,96 & $0,81 * * 3$ & $0,77^{* *}$ \\
\hline Bois de tronc $\ldots \ldots \ldots \ldots$ & 1,05 & 0,97 & 1,14 & 1,01 & 0,84 & 0,89 \\
\hline Ecorce de tronc (écorce + liber) & 1,09 & 1,04 & 1,12 & 0,92 & 0,69 & $0,76 *$ \\
\hline Racines ........ & 1,02 & 0,83 & 1,28 & 0,85 & 0,87 & $0,24 * *$ \\
\hline
\end{tabular}

Valeur des coefficients b de la régression :

$\log$ Minćralomasse $=\log a+b \log$ Biomasse

* Coefficient différent de l'unité au seuil de probabilité de 0,05

* Coefficient différent de l'unité au seuil de probabilité de 0,01 . 
Le cas du phosphore est particulièrement intéressant à considérer puisque cet élément fait l'objet dans la zone étudiée d'un apport systématique en fertilisation à l'installation du peuplement. La figure 1 présente les deux relations valables pour le phosphore dans les aiguilles et dans les branches. Dans le premier cas "l'efficience » ne dépend pas de la vigueur, dans le deuxième, elle s'améliore avec elle. Cette remarque correspond au classique effet de dilution des éléments nutritifs dans les arbres ayant la plus forte croissance (VAN DEN DRIESSCHE, 1974).

\section{PHOSPHORE}

Bronches

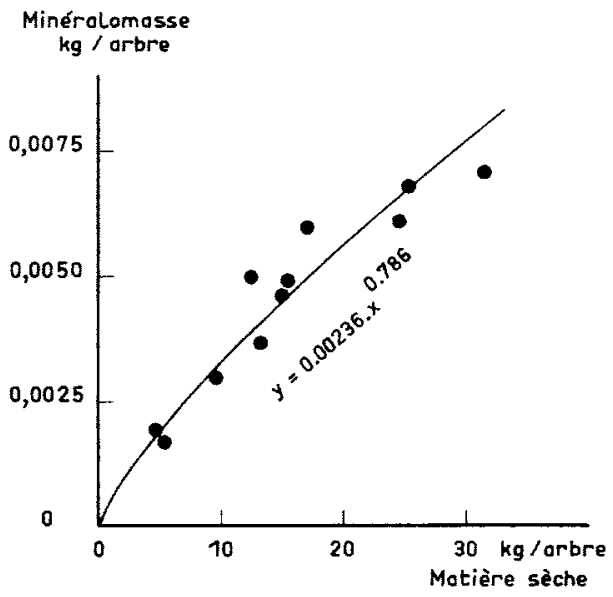

Aiguilles

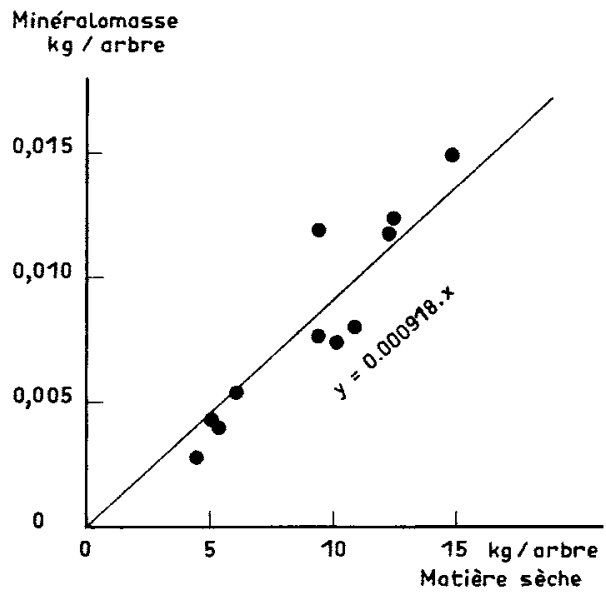

FIG. 1

Relations minéralomasse-biomasse au niveau individuel. Exemple du phosphore dans la cime.

Relationships between biomass and mineral content for each tree. Exemples for phosphorus in the crown (branches and needles).

\subsection{Les concentrations}

D’une manière générale pour un élément donné et pour un compartiment quelconque, une minéralomasse est la résultante de deux composantes multiplicatives : une biomasse et une concentration. La variabilité de la première a été étudiée précédemment (Lemoine et al., 1986). Nous traitons ici des variations de la seconde, et ceci à deux niveaux, celui du compartiment - examen des profils verticaux de concentrations — et celui de l'arbre — variations selon les individus.

\subsection{Profils verticaux moyens}

\subsection{La couronne vivante}

On étudie, selon la position du verticille par rapport au sommet de l'arbre, la variation de concentration d'un élément dans un compartiment (partie de branche) d'âge $A$. 
A ZOTE
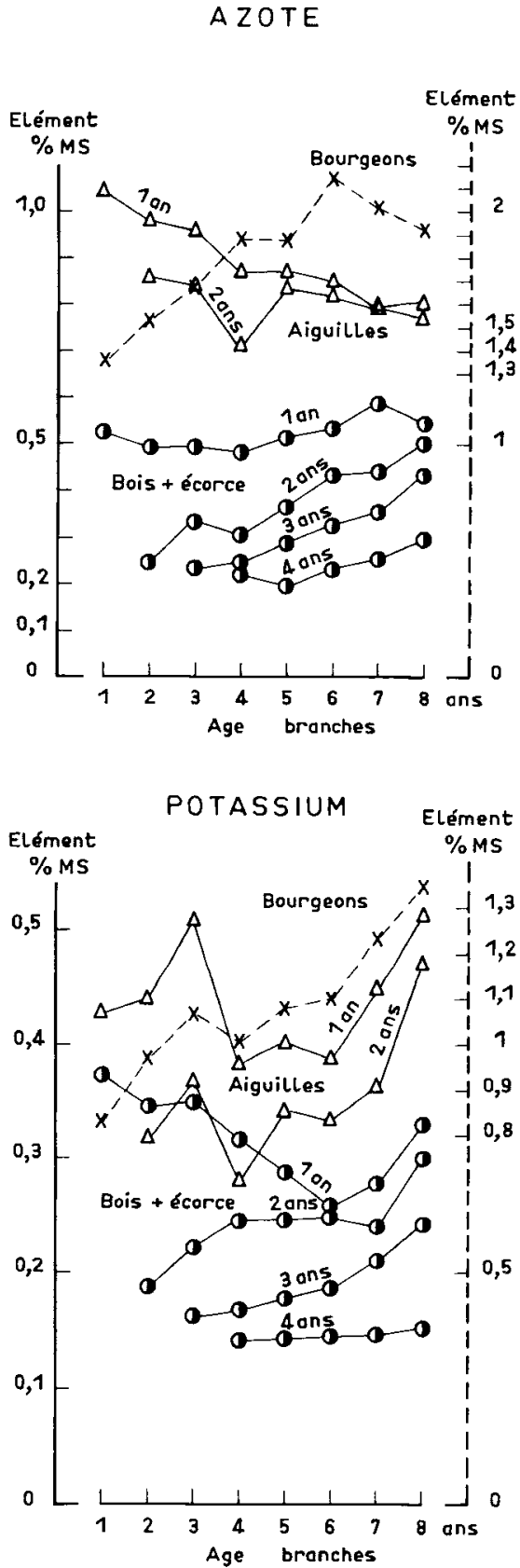

PHOSPHORE

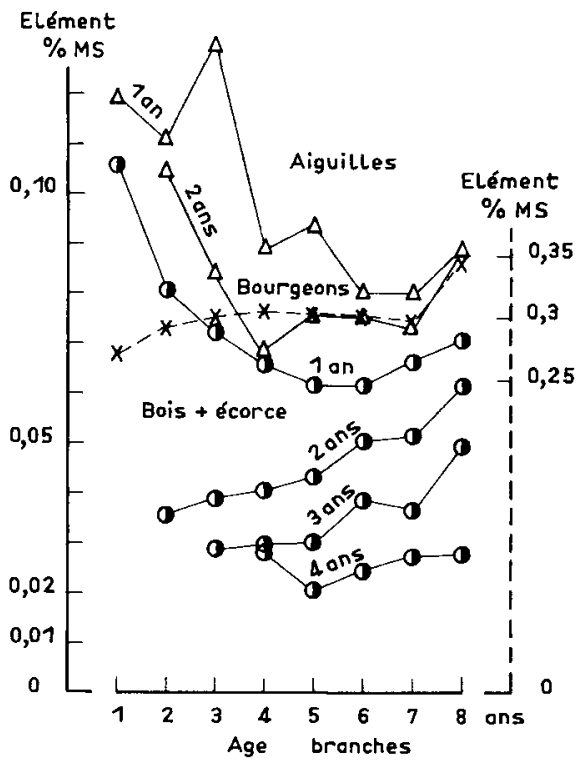

$x---x$ Bourgeons

$\Delta$-.. $\Delta$ Aiguilles

- Bois + écorce

FIg. 2

Concentrations moyennes des éléments nutritifs des branches. Effets de l'âge de la branche et du rameau (moyennes de 8 arbres).

Mean concentration of bioelements in the branches: age effect. 
Les principaux résultats font l'objet de la figure 2. On peut déterminer plusieurs types de profils selon l'élément et le compartiment. A titre d'exemple, du haut vers le bas de l'arbre les concentrations peuvent :

- diminuer comme c'est le cas pour le phosphore des rameaux de un an, l'azote des feuilles de un an...

- rester constantes, pour l'azote des feuilles de deux ans, le phosphore des bourgeons...

- augmenter pour le potassium des rameaux de deux ans, lazote des bourgeons.

Ainsi que l'ont montré plusieurs auteurs, citons Wells \& Metz (1963) pour Pinus taeda L., Magdwick (1964) pour Pinus resinosa (Ait.), Morrison (1972) pour Pinus banksiana Lamb. et Ibid (1984) pour Acer saccharum Marsh. et Betula aleghaniensis L. et ceci uniquement pour les feuilles, il est courant d'observer des comportements opposés dans la distribution des bioéléments dans la couronne des arbres.

De nombreux paramètres sont à prendre en compte tels l'âge de la branche, la durée de vie des aiguilles, l'activité photosynthétique diminuant fortement vers le bas de la cime en peuplement fermé. Ce dernier paramètre provoque soit une diminution des concentrations des aiguilles (effet de dilution pour $\mathrm{N}$ et $\mathrm{P}$ ), soit au contraire une augmentation (effet de concentration pour $\mathrm{K}$ et $\mathrm{Na}$ ) ou encore aucun effet $(\mathrm{Ca}$ et $\mathrm{Mg}$ ). L'influence de la position de la branche sur la composition du «bois » (bois + écorce + liber) des rameaux est généralement semblable à celle observée pour les aiguilles sur les rameaux de 1 an, mais par contre correspond systématiquement à une augmentation des concentrations quand on pénètre dans la couronne pour les rameaux de $2,3,4$ ans et plus; cette influence s'atténue avec l'âge.

Afin de clarifier l'effet de la position du verticille sur les variations de concentrations, on procède à une étude globale de ces variations en retenant : i) 25 variables obtenues en combinant les éléments $\mathrm{N}, \mathrm{P}, \mathrm{K}, \mathrm{Ca}$ et $\mathrm{Mg}$ avec les compartiments feuilles de un an ou de deux ans, bourgeons, bois d'un ou de deux ans ; ii) 7 niveaux du facteur verticille; iii) 8 arbres parmi les 11 analysés de façon à disposer d'un nombre suffisant de niveaux du facteur verticille ( 7 niveaux).

On utilise l'analyse discriminante. On obtient deux axes canoniques significatifs aux pourcentages de discrimination respectifs de 72,5 et 14,4 . La figure 3 présente le profil multivariate à deux composantes des verticilles ainsi que la liaison variables-composantes.

La première composante s'identifie essentiellement à plusieurs variables auxquelles elle est corrélée soit positivement, tels l'azote et le phosphore des feuilles de un an, le magnésium des rameaux de un an, soit négativement, tels le calcium et le potassium des rameaux de deux ans et le calcium et l'azote des bourgeons.

La deuxième composante s'identifie essentiellement au potassium des feuilles de un an. Les autres variables ne montrent pas de liaison privilégiée avec ces composantes.

La première composante fait état d'un profil de variations monotones des concentrations le long de la cime; la deuxième composante complète ces variations en introduisant un minimum pour le verticille de 4 ans. 


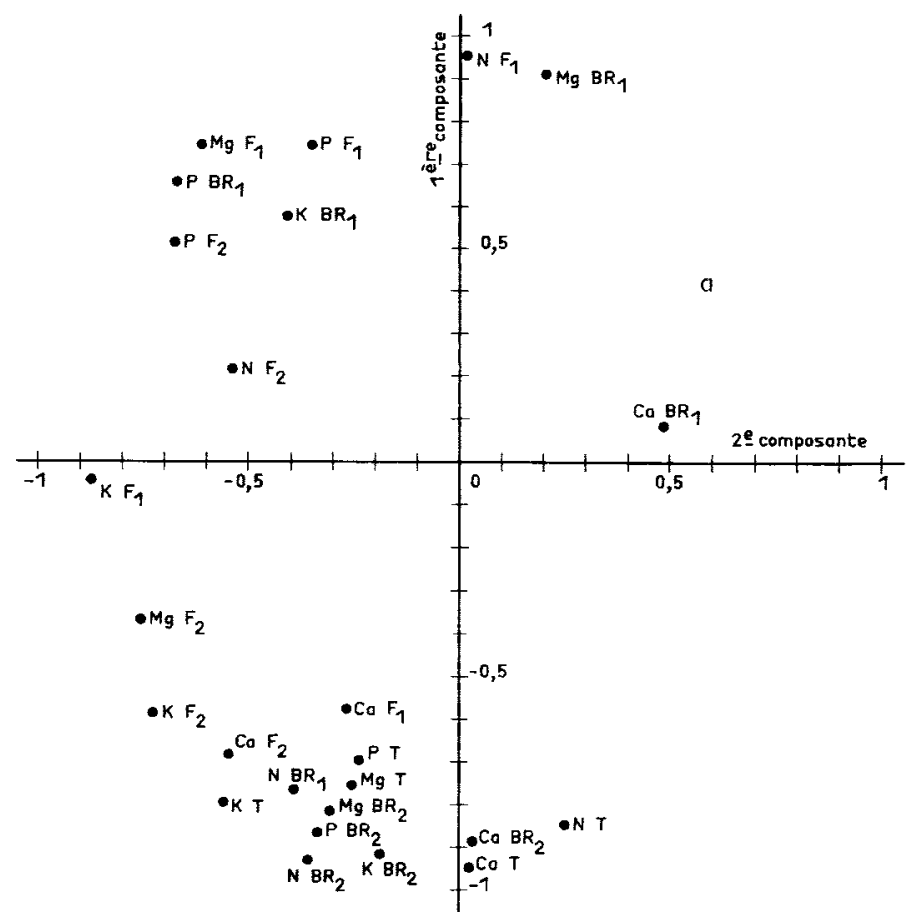

Fig. 3

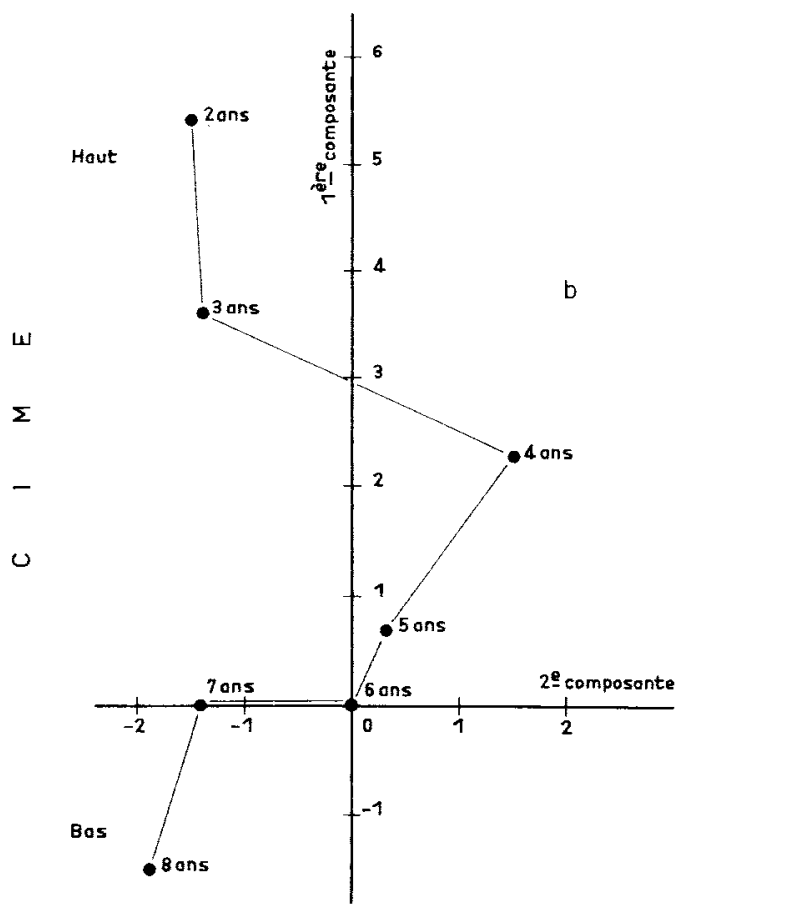


On peut donc formuler les remarques suivantes :

Le premier phénomène traduit l'ensemble des variations continues, le long de la cime, des combinaisons élément-compartiments (nature et âge) : par exemple : augmentation, du haut vers le bas de tous les éléments dans le bois de deux ans de la branche et diminution de certains éléments dans certains compartiments (azote, phosphore et magnésium dans les aiguilles d'un an...). Le deuxième phénomène traduit essentiellement le comportement spécifique des verticilles du milieu de la cime, dû presque exclusivement au potassium dans les aiguilles d'un an : la concentration minima à ce niveau coïncidant avec la biomasse foliaire maxima (LEMOINE et al., 1986) fait penser à un phénomène de dilution.

\subsection{Le tronc}

On étudie, selon le niveau h au-dessus du sol, la variation de concentration d'un élément dans le bois et l'écorce (écorce + liber).

On ne considère que le profil moyen de 7 arbres sur les 11 analysés, afin de disposer de 6 niveaux, les valeurs prises par h sont $0,2,4,6,8$ et $10 \mathrm{~m}$. Les profils font l'objet de la figure 4 . On en distingue deux types selon le compartiment étudié :

- le bois: les concentrations diminuent du haut jusqu'au quart inférieur et réaugmentent dans le bas de l'arbre ;

- l'écorce (écorce + liber) : ces phénomènes sont plus accentués; de plus la courbe présente un point d'inflexion vers le milieu de la cime.

Ces observations concordent pour le bois avec celles de MaLkönen (1974) faites sur Pin sylvestre, par contre, elles sont opposées pour l'écorce.

L'analyse factorielle discriminante a été utilisée pour obtenir une représentation synthétique de la variation des concentrations le long du tronc. Cette analyse est réalisée à partir : i) des 10 variables obtenues en combinant les éléments majeurs $\mathrm{N}, \mathrm{P}$, $\mathrm{K}, \mathrm{Ca}$ et $\mathrm{Mg}$ et les compartiments bois et écorce, ii) des 6 valeurs du facteur niveau dans le tronc $(\mathrm{h})$ variant de 0 à $10 \mathrm{~m}$.

FIG. 3

Concentration des bioéléments dans les branches, variabilité entre verticilles.

Analyse Factorielle Discriminante ( 25 variables éléments $\times$ compartiments) 7 verticilles de 2 à 8 ans.

a) Trace des variables sur le plan des deux premiers axes.

b) Projection des moyennes par verticille sur le même plan.

Nutrient concentrations in the branches. Discriminant factorial analysis. 

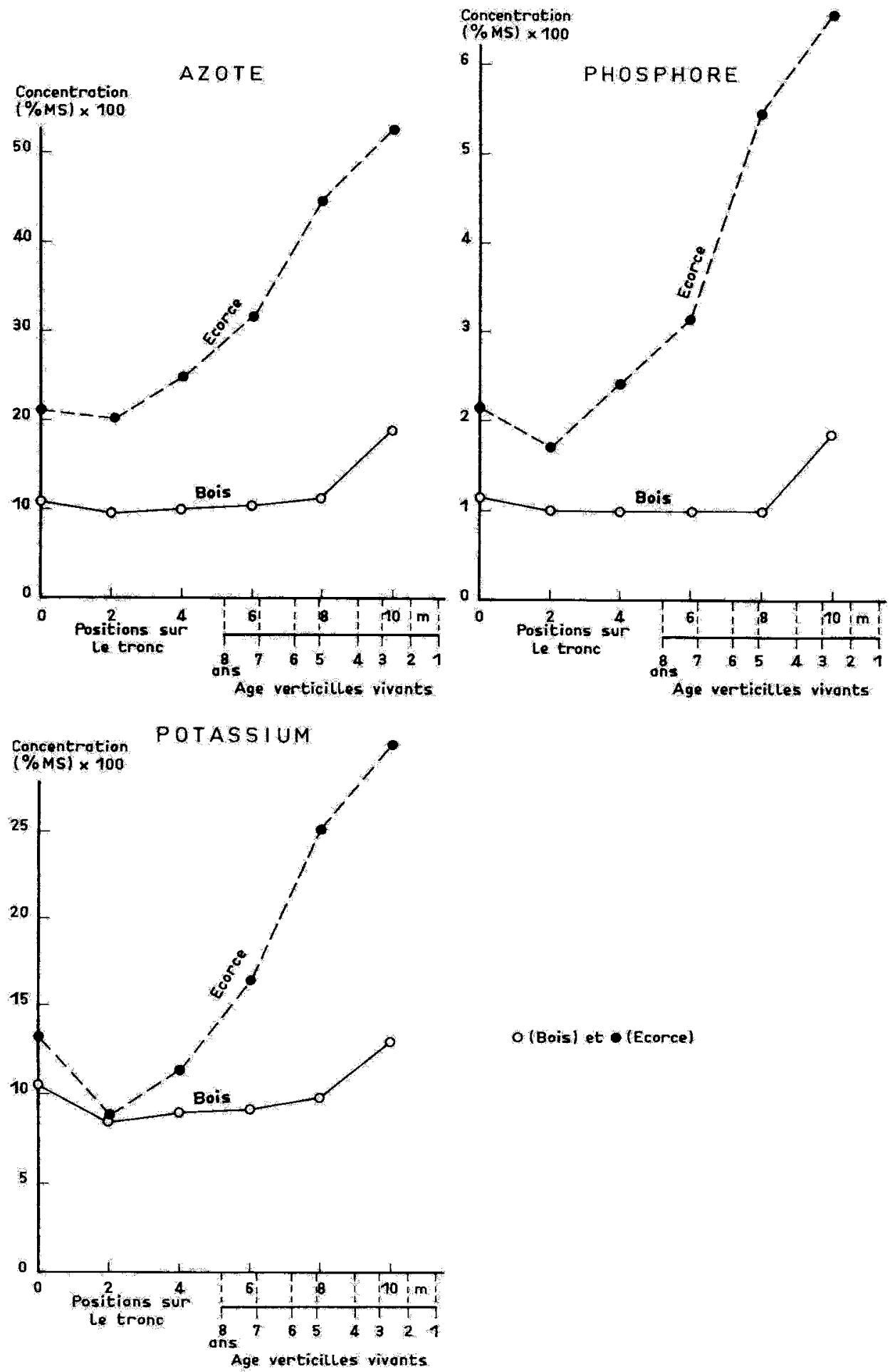

FiG. 4

Variation des concentrations le long du tronc (moyenne de 7 arbres).

Distribution of nutrient concentration in the trunk (mean value for 7 trees). 
On obtient deux axes canoniques significatifs aux pourcentages de discrimination respectifs de 83,2 et 13,5 . La figure 5 présente le profil de variation multivariate à deux composantes du facteur niveau.
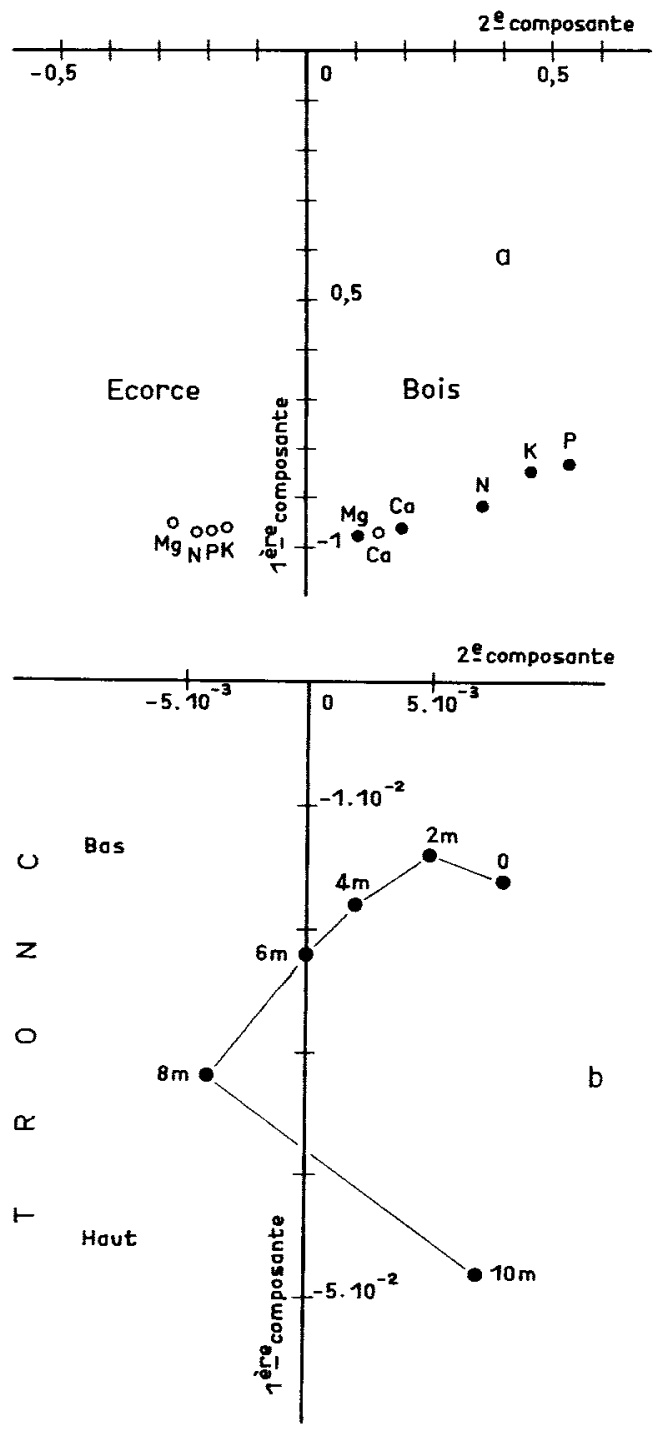

Fig. 5

Concentration des bioéléments dans le tronc: variabilité entre les niveaux.

Analyse Factorielle Discriminante ( 8 variables : combinaison éléments $\times$ compartiments), 6 niveaux. a) Trace des variables sur le plan des deux premiers axes.

b) Projection des moyennes par niveau sur ce même plan.

Nutrient concentration in the trunk : discriminant factorial analysis. 
Les résultats montrent que :

- la première composante est commune à tous les éléments du bois et de l'écorce. Ses variations expriment que du haut vers le bas du tronc toutes les concentrations suivent la même loi de distribution: décroissance forte du haut jusqu'à $2 \mathrm{~m}$ puis remontée faible mais systématique vers le bas du tronc;

- la deuxième composante exprime les modalités spécifiques au bois par rapport à la loi générale. Elles ont trait à l'augmentation brutale des concentrations de $\mathrm{N}, \mathrm{P}, \mathrm{K}$, à partir de $8 \mathrm{~m}$ jusqu'au sommet du tronc ;

- le profil multivariate des données est ainsi curvilinéaire.

Les profils de distribution propres à chaque élément de chaque compartiment ainsi que le profil synthétique issu de l'analyse des données montrent clairement à la fois la forte interdépendance des éléments minéraux, mais aussi des compartiments du tronc bois et écorce.

Concernant les éléments, on retrouve dans cette analyse des données quantitatives la notion d'équilibre entre les éléments (Hel..er, 1974).

\subsection{La variabilité individuelle}

L'étude des profils moyens de distribution des différents éléments minéraux dans l'arbre ne rend certainement pas compte de la totalité de la variabilité observée. En effet, les biomasses et les modalités de croissance (Lemoine et al., 1986) d'une part, et les minéralomasses (cf. \$ 3.122.) d'autre part varient de façon importante en fonction des individus au sein du peuplement.

\subsection{La couronne vivante}

La variation des concentrations en bioéléments dans les sous-compartiments de couronne de chaque arbre échantillonné a été étudiée. Les sous-compartiments retenus correspondent aux accroissements annuels de chaque verticille vivant.

L'analyse statistique porte sur les 25 variables retenues pour l'étude du profil de la cime et ceci pour les verticilles âgés de 2 à 6 ans afin de pouvoir traiter les onze arbres disponibles.

On utilise d'abord l'analyse de variance à un facteur qui rend possible l'analyse discriminante (on ne dispose que d'une branche par combinaison arbre-verticille).

Les tests F univariates montrent que ces arbres, ou populations de branches ( 6 branches par arbre), diffèrent les uns des autres pour presque toutes les variables à l'exception du phosphore des aiguilles de 1 et 2 ans, du calcium dans les sections de branches de 2 ans et du magnésium des bourgeons.

L'analyse discriminante fait ressortir quatre axes significatifs aux pourcentages de discrimination respectifs de 46,6 - 18,5 - 13,6 - 7,8. On identifie respectivement chacun des axes aux éléments azote, calcium, potassium et magnésium, et ceci généralement pour les cinq compartiments.

L'effet arbre est donc composite car chaque élément minéral donne lieu à un type de variation. Cet effet composite est synthétisé par des tests de similarité donnant lieu à l'établissement d'un dendrogramme (fig. 6). Pour un seuil de similarité donné, certains arbres se trouvent rassemblés dans des groupes, d'autres pas. Par exemple, au 
seuil de similarité de 0,87 on peut constituer trois groupes différents d'arbres identiques $\left(\mathrm{n}^{\circ} 11,15\right.$ et $4-\mathrm{n}^{\circ} 18$ et $6-\mathrm{n}^{\circ} 20$ et 7$)$ et quatre groupes monoarbres différents. Au seuil de similarité de 0,716 on peut admettre que tous les arbres qui constituent alors un seul groupe, ont des concentrations similaires.

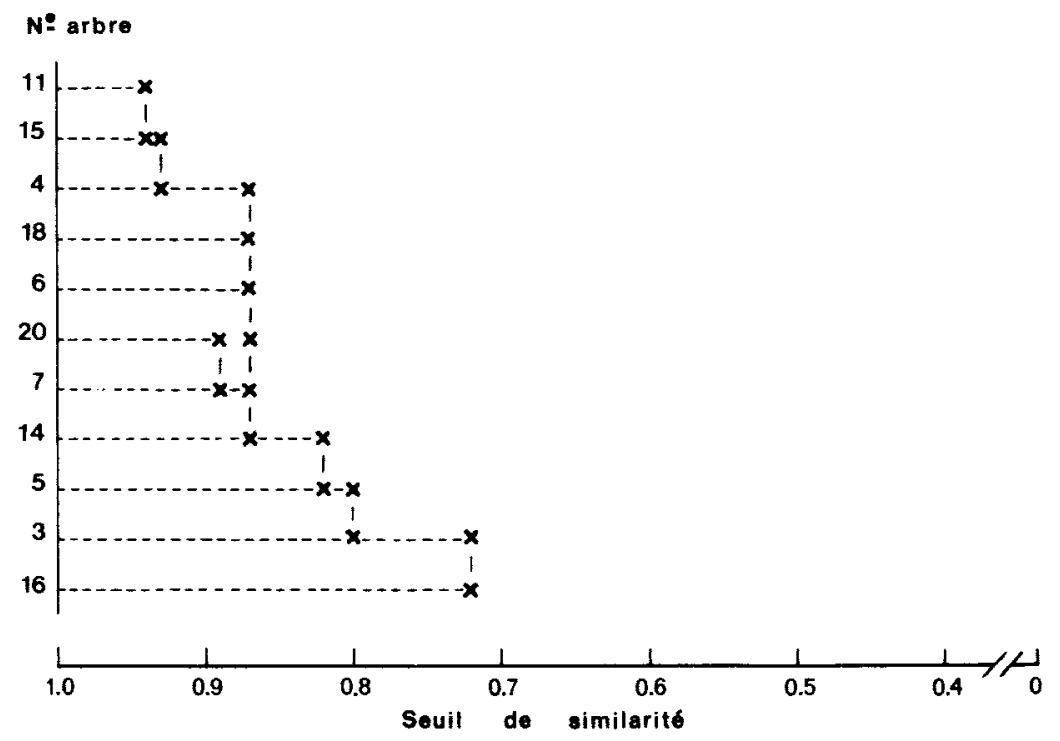

Fig. 6

Concentrations des bioéléments dans la cime: analyse des données sur l'effet arbre (11 arbres, 5 verticilles); 25 variables: 5 éléments ( $N, P, K, C a, M g)$ $\times 5$ compartiments $\left(F_{A}, F_{B}, B G, B A, B B\right)$. Dendrogramme.

Dendrogram showing the tree effect on the distribution of nutrients in the crown.

\subsection{Le tronc}

Parmi les 10 combinaisons possibles entre les cinq éléments, $\mathrm{N}, \mathrm{P}, \mathrm{K}, \mathrm{Ca}$ et $\mathrm{Mg}$ et les deux compartiments bois et écorce, on n'en retient que 8 car le phosphore et le magnésium du bois ne varient apparemment pas d'un arbre à l'autre (le seuil de détection des méthodes analytiques utilisées est en cause).

Afin de pouvoir traiter les 11 arbres, on ne retient que les 5 niveaux étagés de 0 à $8 \mathrm{~m}$ au-dessus du sol. Là encore, on n'utilise que l'analyse de variance à un facteur car on ne dispose que d'une détermination par combinaison arbre-niveau. L'analyse discriminante devient ainsi possible.

Les tests F univariates sont ainsi ( $\mathrm{ddl}=10$ et 44$)$

$\begin{array}{lccccc} & \mathrm{N} & \mathbf{P} & \mathbf{K} & \mathrm{Ca} & \mathrm{Mg} \\ \text { Bois } & 3,01^{* *} & - & 3,86^{* *} & 1,00 \mathrm{NS} & - \\ \text { Ecorce } & 0,77 \mathrm{NS} & 0,45 \mathrm{NS} & 1,09 \mathrm{NS} & 1,17 \mathrm{NS} & 1,32 \mathrm{NS}\end{array}$

Les seuls effets arbre significatifs mis en évidence par l'analyse de variance ont trait à l'azote et au potassium dans le bois. 
L'analyse discriminante fait ressortir trois axes canoniques significatifs aux pourcentages de discrimination respectifs de 37,12 - 26,8 et 18,1. On identifie aisément le premier axe aux effets arbre relatifs au potassium, le second axe à l'azote et le troisième axe à une combinaison de variables opposant le potassium du bois au magnésium de l'écorce.

Ces effets arbre sont synthétisés par le dendrogramme de la figure 7. Au seuil de similarité de 0,82 on ne peut constituer qu'un seul groupe d'arbres identiques $\left(\mathrm{n}^{\circ} 4\right.$ et 6) et 9 groupes monoarbres différents. Il faut descendre jusqu'au seuil de similarité de 0,352 pour ne constituer qu'un seul groupe d'arbres.

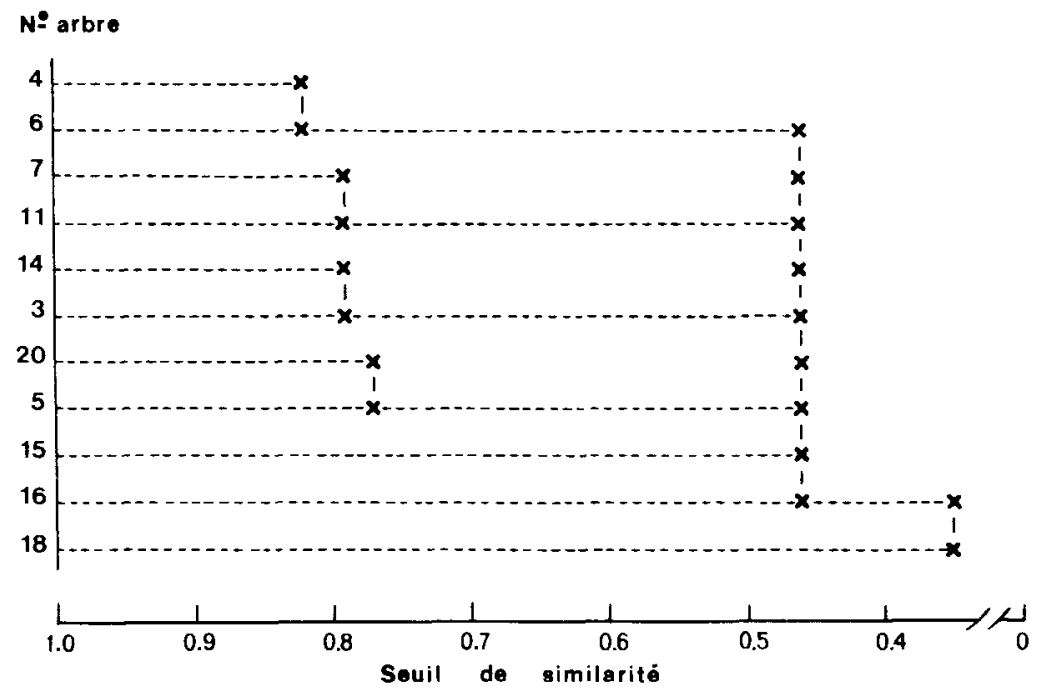

FIG. 7

Concentrations des bioéléments dans le tronc: analyse de donnée sur l'effet arbre (11 arbres - 5 niveaux) 8 variables (éléments $\mathrm{N}, \mathrm{K}, \mathrm{Ca}$

ou N,P, K, Ca, Mg $\times 2$ compartiments Bois-Ecorce). Dendrogramme.

Dendrogram showing the tree effect of the distribution of nutrients in the trunk.

\subsection{Relations avec la croissance}

Nous rappelons certains résultats acquis précédemment (LEMoINE et al., 1986) :

- l'analyse de la croissance en section dans le bas du tronc - surface des cernes successifs - révèle que les accroissements finaux du stade 12-16 ans ne sont presque pas corrélés avec les accroissements initiaux du stade 3-7 ans ;

- les biomasses foliaire et ligneuse de la couronne sont corrélées non seulement avec les accroissements du stade final mais aussi avec ceux du stade initial;

- il s'ensuit donc que l'on ne peut caractériser que très imparfaitement la croissance au niveau individuel en retenant pour seul critère la dimension acquise, soit la surface terrière à 16 ans (ou g16). On utilisera ici comme caractéristique de la croissance les accroissements à 16 et 7 ans, soit Ig16 et Ig7. 
On a vu ci-dessus que les 11 arbres étudiés différaient significativement les uns des autres par bon nombre de concentrations ayant trait à certains éléments minéraux et compartiments de biomasse. Ces variations inter-arbres peuvent être représentées globalement par les composantes des deux analyses discriminantes faites pour la cime et le tronc. On a calculé les corrélations entre les composantes et les accroissements en section $\operatorname{Ig} 7$ et Ig16. La relation entre la première composante du tronc (liée au potassium) et l'accroissement à 16 ans est la plus nette (tabl. 4).

\section{TaBleau 4}

Concentration, variations inter-arbres.

Corrélations entre les composantes des analyses discriminantes

et les accroissements en section 197 et 1916 , à 7 et 16 ans.

Correlations between the principal components calculated for nutrient concentrations and cross sectionnal increment at ages 7 and 16.

\begin{tabular}{c|c|c|c|c}
\hline \hline \multirow{2}{*}{$\begin{array}{c}\text { Accroissements en section } \\
\text { dans le bas du tronc }\end{array}$} & \multicolumn{4}{|c}{$\begin{array}{c}\text { Composantes } \mathrm{C} \\
\text { des analyses discriminantes }\end{array}$} \\
\cline { 2 - 5 } & \multicolumn{2}{|c|}{ Cime } & \multicolumn{3}{|c}{ Tronc } \\
\cline { 2 - 5 } & $\mathrm{C}_{1}$ & $\mathrm{C}_{2}$ & $\mathrm{C}_{1}^{\prime}$ & $\mathrm{C}_{2}^{\prime}$ \\
\hline $\operatorname{Ig} 7$ & 0,471 & 0,232 & $0,636^{*}$ & $-0,106$ \\
$\operatorname{Ig} 16$ & 0,053 & 0,117 & $0,757^{* *}$ & $-0,392$ \\
\hline
\end{tabular}

La variabilité individuelle au sein du peuplement est donc complexe. Elle concerne la vigueur, l'allure de la croissance et la concentration en certains éléments nutritifs. Elle mérite donc une interprétation fonctionnelle qui sera présentée dans un autre article.

\section{Conclusion}

Les recherches entreprises dans un jeune peuplement de Pin maritime de lande humide conduisent aux conclusions suivantes:

1) L'étude de la distribution des éléments minéraux et le passage à la minéralomasse d'un peuplement forestier nécessitent un investissement important qu'il est nécessaire d'optimiser. Les onze arbres, répartis dans un échantillonnage stratifié, utilisés dans cette étude conduisent à une erreur tout à fait acceptable sur la détermination des minéralomasses. En effet, les erreurs à craindre sont en général inférieures à 10 p. 100 pour les compartiments les plus importants èt pour les éléments majeurs considérés. Cet échantillonnage permet de plus de déceler une variabilité individuelle.

Au total, il semble difficile de réduire l'échantillonnage de façon significative si l'on conserve les deux objectifs sus-cités. On rejoint les conclusions de Millier et al., 1985 en notant dans cette étude une variabilité inférieure à celle observée dans les taillis simples. 
2) La réalité de l'erreur ainsi déterminée n'a de sens que dans le contexte d'une méthodologie particulière. Dans le cas de compartiments très pauvres en certains éléments, tel le bois de tronc, la méthode utilisée donne des résultats proches de la limite de détection pour le phosphore et le magnésium. Il ressort que pour l'objectif principal (estimation de la minéralomasse) la méthode est bien adaptée, par contre pour apprécier la variabilité individuelle au niveau des troncs il faudrait utiliser des méthodes plus performantes.

3) La variation des concentrations des bioéléments dans les différents compartiments étudiée par l'analyse factorielle discriminante (AFD) permet de donner pour la couronne et le tronc des profils synthétiques interprétables sur le plan biologique. Dans le tronc, les variations des différents éléments sont parallèles et corrélées positivement. On peut émettre l'hypothèse qu'elles sont le reflet de mécanismes où un élément n'a pas une influence par lui-même mais en référence à tous les autres éléments. Dans ce compartiment, la distribution serait liée d'une part à l'âge moyen des accroissements à un niveau donné et d'autre part à la proximité des zones de prélèvement (racines) ou de redistribution (nappe foliaire). Dans les cimes, les variations sont plus complexes avec des corrélations positives ou négatives entre les éléments d'un même ou des différents sous-compartiments. Dans les aiguilles, la liaison avec la distribution verticale de la masse foliaire est évidente.

Une modélisation de la distribution des éléments, ainsi qu'une étude des corrélations avec les accroissements du tronc devrait permettre d'étayer une interprétation fonctionnelle des répartitions. Cette approche fera l'objet d'un rapport séparé.

4) L'analyse de la variabilité montre que l'effet arbre n'est pas négligeable. Il varie en intensité avec le compartiment : il est plus fort pour le tronc que pour la couronne.

On a relevé des variations individuelles des concentrations très importantes pour le tronc, sensibles pour la cime. Quelle est la cause de ces variations ? On constate déjà que certaines peuvent être mises en relation avec la dynamique de croissance.

Deux hypothèses, non exclusives, peuvent être formulées :

- des variations pédologiques importantes ont été relevées dans le site même d'étude (Courcoux, 1982 et Brossard, 1986) ; elles pourraient créer des différences entre les microstations explorées par les divers arbres;

- une variabilité génétique intra-provenance peut être envisagée ; une étude a été entreprise dans ce sens. Elle vise dans un premier temps à comparer les concentrations de génotypes très différents (pins maritime de races corse et landaise plantés dans la même parcelle).

5) L'évaluation de la minéralomasse du peuplement montre que le Pin maritime possède un rendement élevé des éléments biogènes (biomasse produite/éléments consommés). La frugalité de cette espèce est la plus nette pour le phosphore et le calcium. Il sera intéressant de vérifier si les lois observées dans cet exemple se maintiennent ou non dans des peuplements d'âge différent et/ou situés dans d'autres conditions écologiques.

Reçu le 21 janvier 1987.

Accepté le 10 décembre 1987. 


\section{Summary \\ The qualitative and the quantitative distributions of bioelements in a young stand of Pinus pinaster (Ait.)}

This paper is the second part of the work presented earlier in this issue (LemoINE et al., 1986). The aim of the first paper was to determine the biomass distribution in a young stand (16 years old) of Pinus pinaster (Ait.) situated in the S.W. of France (Landes de Gascogne) which received mineral fertilizer when planted.

The results in this paper concern the distribution of major elements $(\mathrm{N}, \mathrm{P}, \mathrm{K}, \mathrm{Ca}$ and $\mathrm{Mg})$ in the same stand.

Two kinds of results are presented :

1) These concerning the qualitative distribution (concentrations) of bioelements in the various components of trees.

In the living crown, the distribution of elements varies with the components (branches or needles), with their age and with the relative position from the apex. Discriminant factorial analysis (DFA) gives a synthetic profile which shows the main characteristics of this distribution of the continuous vertical variation of elements (positive or negative) and the singular behaviour of the whorl situated in the middle of the crown which corresponds to the area of maximum needle production.

In the trunk, the bioelement distribution is clearer and general trends are similar in the wood and in the bark. The two significant axis of DFA show a synthetic profile whose shape is linked to a general increase in element concentration with position in the trunk, and to the presence of two particular points: a minimum of concentration values at two meters from the ground and an inflexion point in the upper part of the trunk due to changes in bark concentration.

Apart from considering the mean profiles, individual variations have been studied. Divergences from the general law are important and can be evaluated very well by the similarity threshold test, calculated for trees.

Variance analysis shows that the composition of all the tree components has been significantly affected by the tree effect. There are some direct relationships with the trunk increments: trunk composition is related to the present trunk increment. It appears that the chemical composition of the various tree components is not controlled by the same factors.

2) A quantitative evaluation of the mineral content of the stand shows clearly that the production of dry matter in relation to the phosphorus and calcium used is highly efficient.

We are now trying to model these mineral distributions to give a functional interpretation of our results. We have to verify whether the laws of nutrient distribution defined for this stand are applicable or not to other stands of different ages or in different ecological situations.

Key words: Pinus pinaster Ait., nutrients, tissue concentrations, mineral content.

\section{Références bibliographiques}

AlbAN D.H., 1985. Seasonal changes in nutrient concentration and content of Aspen Suckers in Minnesota. Forest. Sci. 31 (3), 785-794.

Baradat PH., 1980. L'« O.P.E.P. », INRA, Départ. Forêts, 293 pp.

BASKERVILle G.L., 1972. Use of logarithmic regression in the estimation of plant biomass. Can. $J$. For., 2, 49-53. 
Brossard M., 1986. Effets de la sylviculture moderne sur la répartition, le niveau et l'état du stock organique des sols de la pinède des Landes de Gascogne. Thèse de $3^{\mathrm{c}}$ cycle, Poitiers, 1 vol., 128 p.

Comps B., Gelpe J., Saint Didier J., 1979. Nouvelle typologie des landes de Gascogne. Modèle d'étude sur la commune de Pissos (Landes). Bull. Ecol. t. 10, n' 1, p. 43-54.

Courcoux P., 1982. Approche du cycle biologique d'un ćcosystème à Pin maritime (Pinus pinaster Ait.) en lande humide. D.E.A. Bordeaux, 1 vol., 41 p.

Decourt N., 1973. Tables de production pour les forêts françaises. Ed. E.N.G.R.E.F., 1 vol., $49 \mathrm{p}$.

Decourt N., Lemonne B., 1969. Tables de production provisoires pour le Pin maritime dans le Sud-Ouest de la France. Ann. Sci. Forest., 26 (1), 3-44.

Farrel F.N., Flinn D.W., SQuire R.O., Craig F.G., 1986. Maintenance of productivity of pine monocultures on sandy soils in Southeast Australia. In "Forest site productivity ", SP Gessel ed., Martinus Nijhoff Pub., 1986.

Gelpe J., Lefrou G., Essai de fertilisation minérale sur Pin maritime à Mimizan (Landes). Résultats après la $26^{\mathrm{e}}$ année. Rev. for. fr., 38, (4), 394-400.

Hell.fr H., 1969. Nutrition et métabolisme. Masson Ed.

Le Gigan P., 1979. L'élaboration de la formation du sable des Landes, dépôt résiduel de l'environnement sédimentaire pliocènc-pléistocène centre aquitain. Thèse d'Etat, Université de Bordeaux I, 429 p., 100 fig., 9 photos.

Lemoine B., Gelpe J., Ranger J., Nys C., 1986. Biomasse et croissance du Pin maritime. Etude de la variabilité dans un peuplement de 16 ans. Ann. Sci. For. 1986, 43 (1), 67-84.

MAGDwick H.A.I., 1964. Variations in the chemical composition of red pine leaves : a comparison of well-grown and poorly grown trees. Forestry, 37 (1), 87-94.

MALKönen E., 1974. Annual primary production and nutrient cycle in some scots pine stands. Communicationes Instituti Forestalis Fenniae 84, 5, 1 vol., 87 p.

Millier C., Nys C., Ranger J., 1986. L'échantillonnage dans l'étude de la minéralomasse forestière. L'exemple des taillis ardennais. Ann. Sci. Forest., 43 (2), 165-178.

MorRisson I.K. 1972. Variation with crown position and leafe age in content of seven clements in leaves of Pinus banksiana Lamb. Can. J. For. Res., 2, 89-94.

MORRISSON I.K. 1985. Effect of crown position of foliar concentrations of 11 elements in Acer saccharum and Betula alleghaniensis trees in a till soil. Can. J. For. Res. Vol. 15, p. 179-183.

Righi D., 1979. Genèse et évolution des podzols et sols hydromorphes des landes du Médoc. Thèse d'Etat, Université de Poitiers, I vol., 144 p.

SAint Didier J., 1976. Nature et évolution de la végétation de la grande lande à Pissos. Mémoire E.N.I.T.A. Dijon, 1 vol., 110 p.

SATOO T., 1982. Forest biomass. H.A.I. Magdwick ed., Martinus Nijhoff Pub.

van den Driessche R., 1974. Predicting of mineral nutrient status of trees by foliar analysis. Bot. Rev., 40 (3), 347-394.

Wells C.G., Metz L.S., 1963. Variation in nutrient content of loblolly pine needles with season age soil and position of the crown. Soil Sci. Soc. Am. Proc., 27 (1), 90-93. 